\title{
A general class of additively decomposable inequality measures ${ }^{\star}$
}

\author{
James E. Foster ${ }^{1}$ and Artyom A. Shneyerov ${ }^{2}$ \\ 1 Department of Economics, Vanderbilt University, Nashville, TN 37235, USA \\ (e-mail: fosterje@ctrvax.vanderbilt.edu) \\ 2 Department of Managerial Economics and Decision Sciences, \\ J.L. Kellogg Graduate School of Management, Northwestern University, Evanston, IL 60208, USA \\ (e-mail: a-shneyerov@nwu.edu)
}

Received: October 27, 1997; revised: March 25, 1998

\begin{abstract}
Summary. This paper presents and characterizes a two-parameter class of inequality measures that contains the generalized entropy measures, the variance of logarithms, the path independent measures of Foster and Shneyerov (1999) and several new classes of measures. The key axiom is a generalized form of additive decomposability which defines the within-group and between-group inequality terms using a generalized mean in place of the arithmetic mean. Our characterization result is proved without invoking any regularity assumption (such as continuity) on the functional form of the inequality measure; instead, it relies on a minimal form of the transfer principle - or consistency with the Lorenz criterion - over two-person distributions.
\end{abstract}

Keywords and Phrases: Inequality measures, Theil measures, Variance of logarithms, Generalized entropy measures, Additive decomposability, Functional equations, Axiomatic characterization.

JEL Classification Numbers: C43, D31, D63, O15.

\section{Introduction}

Decomposability of an inequality measure can be extremely useful for analyzing income inequality in a population partitioned according to identifiable characteristics, such as geographic location, race or gender. The failure of the well-known

\footnotetext{
* We thank Tony Shorrocks, the anonymous referees, and the editor for helpful comments. Support from the John D. and Catherine T. MacArthur Foundation through its Network on Inequality and Poverty in Broader Perspective is gratefully acknowledged.

Correspondence to: J.E. Foster
} 
Gini coefficient to have a neat decomposition into "within-group" and "betweengroup" terms accounts for its relatively infrequent use in this type of analysis. In contrast, the generalized entropy class of measures, including the two Theil measures and the (squared) coefficient of variation, was derived specifically for this purpose, and indeed can be axiomatically characterized using a property of "additive decomposability", e.g., see Shorrocks (1980). The variance of logarithms, which is often employed in the evaluation of wage inequality, is also decomposable, but has a slightly different formula based on the geometric rather than the arithmetic mean. The present paper explores a broad framework for inequality decomposition that includes all of the aforementioned measures, and provides a complete characterization of the class of inequality measures exhibiting this general form of additive decomposition.

Many questions relating to economic inequality do not require a measure to be decomposable by population subgroup; in these cases an overall evaluation of inequality is sufficient. However, there are other questions that naturally focus on certain well-defined groups in the population, and call for an assessment of the amount of the total inequality that can be attributed to variation within the groups or, alternatively, differences across the groups. The traditional approach to decomposition is similar to the well-known "analysis of variance," which decomposes overall variance into two terms. The "within-group term" is a weighted sum of variances in the population subgroups, with the weights being the group population share. The "between-group term" is the variance of a "smoothed" distribution, in which group incomes are replaced by group means. So, for example, if the overall distribution comprises the four incomes $(2,4,5,7)$ partitioned into subgroups $(2,4)$ and $(5,7)$, the within-group term would be half the variance of $(2,4)$ plus half the variance of $(5,7)$, while the between-group term would be the variance of the smoothed distribution $(3,3,6,6)$. The variance, however, is not independent of proportional changes in incomes; in other words it is not a "relative" inequality measure.

Theil (1967) introduced two relative inequality measures based on tools from information theory, each of which has an additive decomposition similar to the variance decomposition. The "first" Theil measure uses income-share weights in its within-group term, so that in the above example the weight on the subgroup $(2,4)$ would be $1 / 3$ while the weight on $(5,7)$ is $2 / 3$. The decomposition of the "second" Theil measure uses population shares and, like the variance, assigns both subgroups in the example a weight of $1 / 2$. Bourguignon (1979) proved that among all relative measures that are differentiable, each Theil measure is completely characterized by its respective decomposability property. Foster (1983) showed that in the characterization of the first Theil measure, the regularity property could be replaced by the "transfer principle", which requires a transfer of income from a poorer to a richer person to increase inequality ${ }^{1}$.

The generalized entropy class extends Theil's two measures to an entire family of inequality measures, each exhibiting an additive decomposition analogous

${ }^{1}$ A similar approach is used by Gehrig $(1983,1988)$ in the measurement of industrial concentration. 
to the variance but with the weights in the within-group term being functions of subgroup means and population sizes ${ }^{2}$. Shorrocks (1980) gave a key characterization of this class as essentially the only differentiable measures with such a (weighted) additive decomposition, where weights can be general functions of the subgroup means and population sizes. In subsequent work Shorrocks (1984) showed that the assumption of differentiability can be reduced to continuity ${ }^{3}$.

All of the above work uses the arithmetic mean as the "representative income" in constructing the weights for the within-group term and the smoothed distribution for the between-group term. In contrast, the variance of logarithms employs the geometric mean and, indeed, other forms are also plausible. Blackorby, Donaldson and Auersperg (1981) argue forcefully for the use of a "generalized" or " $q$-order mean" - the $q$ th-root of the average $q$-power income - in decompositions of welfare-based indices. In other words they suggest the use of Atkinson's (1970) specific form of "equally distributed equivalent income" as the representative income in the decomposition formula. So, for example, in evaluating subgroup distributions $(1,9)$ and $(5,5)$, there is no clear reason for requiring between-group inequality to be zero, as would be the case if the arithmetic mean were used. Indeed, there is a real sense in which the average person in $(1,9)$ is worse off than the average person in $(5,5)$ and, if so, this should be reflected in the respective representative income levels. They show that the $q$-order means for $q \leq 1$ fulfill this requirement and that, in fact, they are the only forms of representative income that make sense in their context. Later work by Foster and Shneyerov (1999) derives the $q$-order means from a basic consistency requirement for decompositions called "path independence" and, with the help of continuity, characterizes the single-parameter class of measures implied by this property.

The present paper extends the standard axiom of additive decomposability to allow the use of any given $q$-order mean as representative income in the decomposition formula. The resulting property, which we call "general additive decomposability" (or "GAD") has the following features: (1) Overall inequality is the sum of a within-group and a between-group term; (2) The within-group term is a weighted sum of subgroup inequalities, where the weights depend on the population sizes and the generalized means of the subgroups; (3) The smoothed distribution in the between-group term is based on the population sizes and the generalized means of the subgroups. This clearly includes the traditional form of additive decomposition using the arithmetic mean (satisfied by the generalized entropy measures). In addition, though, it broadens the possible ways that subgroup prosperity is represented in the decomposition, thus admitting other potentially useful decompositions and measures (such as the variance of logarithms).

\footnotetext{
${ }^{2}$ See Shorrocks (1980), Cowell (1980), Cowell and Kuga (1981) and Bourguignon (1979), who introduced this class of measures.

${ }^{3}$ Shorrocks (1984) also showed that the additive form of decomposition can be generalized to "aggregability", which admits more general aggregation formulas relating subgroup data to overall inequality.
} 
This paper offers a complete characterization of the set of relative inequality measures satisfying GAD. Our main result is developed in two stages. First, we show that for relative measures satisfying GAD, the weights must take on a special functional form analogous to the weights employed by the generalized entropy measures, but using a generalized mean in place of the arithmetic mean. Second, we show that the set of relative measures satisfying GAD is a two parameter family that contains inter alia the generalized entropy class, including the two Theil measures and the (squared) coefficient of variation, and the "path independent" class of Foster and Shneyerov (1999), including the variance of logarithms. Our proofs draw upon techniques used by Shorrocks $(1980,1984)$ in inequality measurement, using results from the theory of functional equations found in Aczél (1966) and Aczél and Dhombres (1989).

One interesting feature of our characterization theorems is that we place no regularity assumptions at all on the inequality measures. Non-differentiable measures like the Gini are in principle allowed as well as those exhibiting discontinuous jumps. It turns out that enough regularity arises from the "two-person transfer principle", which requires inequality to rise in the two-income case when the poorer person transfers income to the richer one. Consequently, the characterization yields the conclusions of Bourguignon (1979), Shorrocks (1980) and Foster (1983) when the respective regularity assumptions are replaced by this natural property.

We begin in the next section with the basic definitions that will be used in the paper. Our characterization results are presented and proved in Sect. 3, while the new class of measures is discussed in Sect. 4. Section 5 offers some concluding remarks.

\section{Definitions}

Let $R_{++}$and $N$ denote the positive real numbers and the natural numbers, respectively. A typical income distribution will be represented as a positive vector $x=\left(x_{1}, \ldots, x_{n}\right)$ with arbitrary dimension $n \in N$, where $x_{i}$ is interpreted as the income of person $i$. The overall set of distributions under consideration is given by $D=\cup_{n \geq 1} R_{++}^{n}$. We denote the dimension, the (arithmetic) mean, and the geometric mean of a given distribution $x$ by $n(x), \mu(x)$, and $g(x)$, respectively. The symbol $(x)^{q}$ will be used to represent the distribution obtained from $x$ by raising each income to the $q$ th power. Similarly, $\ln (x)$ and $e^{(x)}$ will denote the distribution whose entries are, respectively, the natural logarithm and the exponential of the entries in $x$.

An inequality measure is a function $I: D \rightarrow R$ which, for each distribution $x$, indicates a level $I(x)$ of inequality in the distribution. Several properties for inequality measures will now be presented, which make use of the following notation and definitions. Denote the unit vector in $R^{n}$ by $u_{n}=(1, \ldots, 1)$. We shall call $x \in D$ completely equal if it is a scalar multiple of $u_{n}$ for some positive integer $n$. We say that $x \in D$ is obtained from $y \in D$ : by a permutation if 
$x=P y$ for some permutation matrix $P$ of appropriate dimension; by a replication if $x=(y, y, \ldots, y)$, i.e., the incomes in $x$ are simply the incomes in $y$ repeated a finite number of times; by a scalar multiple if $x=t y$ for some positive real $t$; by a translation if $x=y+t u_{n}$ for some positive real $t$; and by a regressive transfer if, for some $i$ and $j$ with $x_{i} \leq x_{j}$ we have $y_{i}-x_{i}=x_{j}-y_{j}>0$, while for all $k \neq i, j$ we have $x_{k}=y_{k}$.

For the purposes of this paper, a relative inequality measure is a function $I$ : $D \rightarrow R$ satisfying the following properties: (1) Symmetry: $I(x)=I(y)$ whenever $x$ is obtained from $y$ by a permutation; (2) Replication Invariance: $I(x)=I(y)$ whenever $x$ is obtained from $y$ by a replication; (3) Scale Invariance: $I(x)=I(y)$ whenever $x$ is obtained from $y$ by a scalar multiple; (4) Normalization: $I(x)=0$ if and only if $x$ is completely equal; (5) Two-income Transfer Principle: $I(x)>I(y)$ whenever $x \in R_{++}^{2}$ is obtained from $y \in R_{++}^{2}$ by a regressive transfer.

The first three of these properties ensure that $I$ is a relative measure in the sense that it depends only on the frequencies of the relative incomes, and not on the order of the incomes, the overall number of persons, nor the absolute income levels. Normalization fixes the value at zero exactly when the distribution is completely equal. The final property is a substantial weakening of the well- known transfer principle to distributions of income having only two persons ${ }^{4}$. Notice that we have not assumed the general transfer principle nor do we place any continuity requirements - or any other regularity assumptions - on $I$. The twoincome transfer principle provides sufficient regularity for our characterization results.

Now suppose that the overall distribution $x$ is partitioned into $m \geq 2$ subgroup distributions, say $x^{1}, \ldots, x^{m}$, where $\mu^{j}=\mu\left(x^{j}\right)$ denotes the mean of the $j^{\text {th }}$ subgroup and $n^{j}=n\left(x^{j}\right)$ represents its size. Let $\boldsymbol{\mu}=\left(\mu^{1}, \ldots, \mu^{m}\right)$ and $\mathbf{n}=\left(n^{1}, \ldots, n^{m}\right)$ be the vectors of subgroup means and population sizes, respectively. The decomposition formula will make use of this "subgroup data" $(\boldsymbol{\mu}, \mathbf{n})$ for both the weights employed in the within-group term and the distribution used to define the between-group term. The weighting function $w$ maps each $(\boldsymbol{\mu}, \mathbf{n}) \in R_{++}^{m} \times N^{m}$ to a vector of weights $w(\boldsymbol{\mu}, \mathbf{n})=\left(w^{1}(\boldsymbol{\mu}, \mathbf{n}), \ldots, w^{m}(\boldsymbol{\mu}, \mathbf{n})\right)$, where $w^{j}(\boldsymbol{\mu}, \mathbf{n}) \geq 0$ is the weight on subgroup $j$ 's inequality level $I\left(x^{j}\right)$ in the within-group term. The between-group term is based on the smoothed distribution $\chi(\boldsymbol{\mu}, \mathbf{n})=\left(\mu\left(x^{1}\right) u_{n^{1}}, \ldots, \mu\left(x^{m}\right) u_{n^{m}}\right)$ which replaces the incomes of the $n^{j}$ persons in group $j=1, \ldots, m$ with the subgroup mean.

An inequality measure $I$ is said to be additively decomposable if there exists a weighting function $w(\boldsymbol{\mu}, \mathbf{n})$ such that for any $m \geq 2$ and all $x^{1}, \ldots, x^{m} \in D$ we have

\footnotetext{
${ }^{4}$ The general transfer principle requires a regressive transfer to increase inequality for any sized distribution. In the presence of symmetry it is equivalent to strict Schur-convexity of $I$ over each $n$-person domain. In the context of relative inequality measures, the transfer principle is equivalent to Lorenz consistency, which requires $I$ to follow the Lorenz criterion whenever it applies. See for example Foster (1985). Promislow (1991) introduced (a weak form of) the two-income transfer principle in the context of measures of "unfairness".
} 


$$
I\left(x^{1}, \ldots, x^{m}\right)=\sum_{j=1}^{m} w^{j}(\boldsymbol{\mu}, \mathbf{n}) I\left(x^{j}\right)+I(\chi(\boldsymbol{\mu}, \mathbf{n})) .
$$

In other words, an additively decomposable measure can be expressed as the sum of a within-group term, which itself is a weighted sum of subgroup inequality levels, and a between-group term, which is the inequality remaining in the smoothed distribution ${ }^{5}$. So, for example, if $x=(1,5,3,5)$ is partitioned into $x^{1}=(1,5)$ and $x^{2}=(3,5)$, then the property requires $I(1,5,3,5)=$ $w^{1} I(1,5)+w^{2} I(3,5)+I(3,3,4,4)$.

The second Theil measure $T_{2}(x)=\frac{1}{n(x)} \sum_{i=1}^{n(x)} \ln \frac{\mu(x)}{x_{i}}=\ln (\mu(x) / g(x))$, also known as the mean logarithmic deviation, is additively decomposable with population share weights $w^{j}(\boldsymbol{\mu}, \mathbf{n})=n^{j} / n$, where the total population $n=n^{1}+\cdots+n^{m}$ is clearly a function of $(\boldsymbol{\mu}, \mathbf{n})$. The first Theil measure $T_{1}(x)=\frac{1}{n(x)} \sum_{i=1}^{n(x)} \frac{x_{i}}{\mu(x)} \ln \frac{x_{i}}{\mu(x)}$ also satisfies this property, but with weights $w^{j}(\boldsymbol{\mu}, \mathbf{n})=\left(n^{j} \mu^{j}\right) /(n \mu)$ corresponding to subgroup income shares, where it is likewise clear that the overall mean $\mu=\Sigma_{j}\left(n^{j} \mu^{j}\right) / n$ depends only on $(\boldsymbol{\mu}, \mathbf{n})$. Both measures are part of a single parameter family called the generalized entropy measures, which take the form

$$
I_{c}(x)= \begin{cases}\frac{1}{c(c-1)} \frac{1}{n(x)} \sum_{i=1}^{n(x)}\left[\left(\frac{x_{i}}{\mu(x)}\right)^{c}-1\right] & c \neq 0,1 \\ T_{1}(x) & c=1 \\ T_{2}(x) & c=0\end{cases}
$$

It can be shown that each generalized entropy measure $I_{c}$ has an additive decomposition with weights $w^{j}=\left(n^{j} / n\right)\left(\mu^{j} / \mu\right)^{c}$. Shorrocks $(1980,1984)$ proved an important result, showing that these measures (or their positive multiples) are, in fact, the only continuous, normalized, relative measures that satisfy additive decomposability ${ }^{6}$. The practical impact of this and allied characterization results can be seen in the many studies that have subsequently employed this family of measures ${ }^{7}$.

Another commonly-used measure is the variance of logarithms

$$
V_{L}(x)=\frac{1}{n(x)} \sum_{i=1}^{n(x)}\left[\ln x_{i}-\mu(\ln (x))\right]^{2}=\frac{1}{n(x)} \sum_{i=1}^{n(x)}\left[\ln x_{i}-\ln g(x)\right]^{2}
$$

where $g(x)=\left(\prod_{i=1}^{n(x)} x_{i}\right)^{\frac{1}{n(x)}}$ is the geometric mean of $x$. The variance of logarithms has a decomposition formula of the form

\footnotetext{
5 See Love and Wolfson (1976), Anand (1983), Shorrocks (1988), Cowell (1998), and Foster and Sen (1997) for further discussions of decomposability.

${ }^{6}$ Shorrocks (1980) assumes I to be differentiable; the results from Shorrocks (1984) show that this regularity assumption can be reduced to continuity of $I$, i.e., that $I$ is continuous on each $n$-person slice of $D$. See also Shorrocks (1988).

7 See for example Shorrocks and Mookherjee (1982), Cowell (1984), Tsakloglou (1993), Cowell and Jenkins (1995), and Jenkins (1995).
} 


$$
V_{L}\left(x^{1}, \ldots, x^{m}\right)=\Sigma_{j} w^{j}(\mathbf{g}, \mathbf{n}) V_{L}\left(x^{j}\right)+V_{L}(\chi(\mathbf{g}, \mathbf{n}))
$$

where the geometric means have replaced arithmetic means in the subgroup data. The geometric mean places greater emphasis on the lower incomes of the distribution and consequently offers an alternative "representative income" for use in decompositions. Blackorby, Donaldson and Auersperg (1981) go beyond the use of the arithmetic or geometric mean in decomposition formulas, and recommend the generalized or $q$-order means defined by

$$
\mu_{q}(x) \begin{cases}\left(\frac{1}{n(x)} \sum_{i=1}^{n(x)} x_{i}^{q}\right)^{\frac{1}{q}} & q \neq 0 \\ g(x) & q=0\end{cases}
$$

as subgroup representative incomes. They emphasize the welfare interpretation of $\mu_{q}$ for $q \leq 1-$ it is the "equally distributed equivalent income" function that underlies Atkinson's parametric family of inequality measures - and employ it to represent subgroups in the between-group term. They argue that this is a superior method of analyzing between- group inequality, since the resulting term, "measures differences in the actual economic positions of the subgroups rather than their potential economic positions"

Foster and Shneyerov (1999) likewise consider decompositions based on representative income functions and derive the entire class of $q$-order means from a consistency property they call "path independent decomposability". The resulting class of path independent inequality measures

$$
I_{q}(x)= \begin{cases}\frac{1}{q} \ln \left(\frac{\mu_{q}(x)}{g(x)}\right) & q \neq 0 \\ \frac{1}{2} V_{L}(x) & q=0\end{cases}
$$

includes (half) the variance of logarithms (when $q=0$ ) as well as the second Theil measure (when $q=1$ ). Each $I_{q}$ measure has an additive decomposition analogous to the formulas for $V_{L}$ and $T_{2}$ but employing a generalized mean as a subgroup's representative income.

Additive decomposability has proved to be a useful property for the analysis of inequality by population subgroup. Extending this property to include arbitrary $q$-order means is a natural generalization that encompasses all of the above decompositions and measures. We say that an inequality measure $I$ satisfies general additive decomposability or $G A D$, if there is a $q \in R$ and a weighting function $w\left(\boldsymbol{\mu}_{\mathbf{q}}, \mathbf{n}\right)$ such that for any $m \geq 2$ and all $x^{1}, \ldots, x^{m} \in D$ we have

$$
I\left(x^{1}, \ldots, x^{m}\right)=\sum_{j=1}^{m} w^{j}\left(\boldsymbol{\mu}_{\mathbf{q}}, \mathbf{n}\right) I\left(x^{j}\right)+I\left(\chi\left(\boldsymbol{\mu}_{\mathbf{q}}, \mathbf{n}\right)\right) .
$$

\footnotetext{
${ }^{8}$ Blackorby, Donaldson and Auersperg (1981, p. 666). Note that their decomposition (of Atkinson's measure) has an additional "interaction" term and hence is not a "general additive decomposition" of the form we study here. See also Cowell and Jenkins (1995).
} 
Thus $I$ can be expressed as a weighted sum of subgroup inequality measures plus the inequality in a smoothed distribution, where both the weight and the smoothed distribution depend on subgroup population sizes and $q$-order means. We now turn to a characterization of measures that exhibit this broadened form of decomposition.

\section{Characterizations}

We begin by exploring the possible weighting functions that an inequality measure satisfying GAD might exhibit.

Proposition 1. Let $I$ be an inequality measure satisfying GAD for some $q \in R$. Then there exists $c \in R$, such that for every $m \geq 2$ and all $j=1, \ldots, m$ with $n^{j} \geq 2$, the jth subgroup's weighting function has the form

$$
w^{j}\left(\boldsymbol{\mu}_{\mathbf{q}}, \mathbf{n}\right)=\frac{n^{j}}{n}\left(\frac{\mu_{q}^{j}}{\mu_{q}}\right)^{c},
$$

where $n$ and $\mu_{q}$ are, respectively, the aggregate population size and q-order mean.

Proof. Fix $q \in R$ and $m \geq 2$, and pick any $j=1, \ldots, m$. We first show that whenever $n^{j} \geq 2$, the $j$ th subgroup's weighting function $w^{j}$ can be expressed as a function of the ratio $\left(\mu_{q}^{j} / \mu_{q}\right)$ and the population sizes $n^{j}$ and $n$.

Choose any $\left(\boldsymbol{\mu}_{\mathbf{q}}, \mathbf{n}\right)$ having $n^{j} \geq 2$, and let $x^{1}, \ldots, x^{m}$ and $z^{1}, \ldots, z^{m}$ be two sets of subgroup distributions consistent with $\left(\boldsymbol{\mu}_{\mathbf{q}}, \mathbf{n}\right)$ such that $x^{j}$ and all $x^{k}=z^{k}$ with $k \neq j$ are completely equal, while $z^{j}$ is not completely equal. Then by GAD and normalization, we obtain

$$
I\left(z^{1}, \ldots, z^{m}\right)-I\left(x^{1}, \ldots, x^{m}\right)=w^{j}\left(\boldsymbol{\mu}_{\mathbf{q}}, \mathbf{n}\right) I\left(z^{j}\right) .
$$

Now gathering up the subgroup distributions for $k \neq j$ into a new subgroup distribution $y=\left(z^{1}, \ldots, z^{j-1}, z^{j+1}, \ldots, z^{m}\right)$, we can apply GAD, symmetry and normalization, to get

$$
\begin{aligned}
I\left(z^{1}, \ldots, z^{m}\right) & -I\left(x^{1}, \ldots, x^{m}\right) \\
& =I\left(z^{j}, y\right)-I\left(x^{j}, y\right) \\
& =w^{1}\left(\mu_{q}^{j}, \mu_{q}(y), n^{j}, n(y)\right) I\left(z^{j}\right) .
\end{aligned}
$$

Normalization ensures that $I\left(z^{j}\right) \neq 0$, so that

$$
w^{j}\left(\boldsymbol{\mu}_{\mathbf{q}}, \mathbf{n}\right)=w^{1}\left(\mu_{q}^{j}, \mu_{q}(y), n^{j}, n(y)\right)
$$

follows immediately from (2) and (3). Scale invariance of $I$ further yields

$$
w^{j}\left(\mu_{q}^{j}, \mu_{q}(y), n^{j}, n(y)\right)=w^{1}\left(\mu_{q}^{j} / \mu_{q}, \mu_{q}(y) / \mu_{q}, n^{j}, n-n^{j}\right)
$$


where $n=\Sigma_{k} n^{k}$ and $\mu_{q}=\left(\Sigma_{k}\left(\mu_{q}^{k}\right)^{q} n^{k} / n\right)^{1 / q}>0$. Moreover, since $\mu_{q}(y) / \mu_{q}$ can be expressed as a function of $\mu_{q}^{j} / \mu_{q}, n^{j}$, and $n$, it follows that there exists a function $f(r, a, b)$ such that

$$
w^{j}\left(\boldsymbol{\mu}_{\mathbf{q}}, \mathbf{n}\right)=f\left(\mu_{q}^{j} / \mu_{q}, n^{j}, n\right) .
$$

The next step is to derive the form of $f$.

As $\left(\boldsymbol{\mu}_{\mathbf{q}}, \mathbf{n}\right)$ ranges across all possible configurations of subgroup data with $n^{j} \geq 2$, the variables $a=n^{j}$ and $b=n$ range across all natural numbers satisfying $2 \leq a<b$. The variable $r=\mu_{q}^{j} / \mu_{q}$, on the other hand, traces out the interval $\left(0,(b / a)^{1 / q}\right)$ for $q>0$, the interval $(0, \infty)$ for $q=0$, and the interval $\left((b / a)^{1 / q}, \infty\right)$ for $q<0$. We now show that $f$ satisfies the functional equation

$$
f(r, a, b) f(s, b, d)=f(r s, a, d)
$$

on its domain. ${ }^{9}$ To see this, construct subgroup distributions $x^{1}, x^{2}, x^{3}$ satisfying $\mu_{q}\left(x^{1}\right)=r, \mu_{q}\left(x^{1}, x^{2}\right)=1, \mu_{q}\left(x^{1}, x^{2}, x^{3}\right)=1 / s, n\left(x^{1}\right)=a, n\left(x^{1}, x^{2}\right)=b$, and $n\left(x^{1}, x^{2}, x^{3}\right)=d$, such that each $x^{k}$ is completely equal. Let $z^{1}$ be any distribution that is not completely equal and satisfies $\mu_{q}\left(z^{1}\right)=r$ and $n\left(z^{1}\right)=a$. Applying GAD and normalization yields

$$
I\left(z^{1}, x^{2}, x^{3}\right)-I\left(x^{1}, x^{2}, x^{3}\right)=f(r s, a, d) I\left(z^{1}\right) .
$$

If we apply GAD in two stages rather than one, we obtain

$$
\begin{aligned}
I\left(z^{1}, x^{2}, x^{3}\right) & -I\left(x^{1}, x^{2}, x^{3}\right)=f(s, b, d)\left[I\left(z^{1}, x^{2}\right)-I\left(x^{1}, x^{2}\right)\right] \\
& =f(s, b, d) f(r, a, b) I\left(z^{1}\right),
\end{aligned}
$$

which by $I\left(z^{1}\right) \neq 0$ yields the functional equation (5).

The next step in characterizing $f$ is to show that it has a useful regularity property: for each $a, b$ with $2 \leq a<b$, the function $f(r, a, b)$ is bounded on an interval containing $r=1$ in its interior. In fact, it is enough to show this for the function $\bar{f}(r)=f(r, 2,4)$ since one can apply (5) with $r=1$ or $s=1$ as needed to obtain $f(r, a, b)=\alpha \bar{f}(r)$ for some $\alpha>0$. So consider the distribution $(1, s, t, s t)$ for any $s, t>0$. Clearly $\mu_{q}(1, s, t, s t)=\mu_{q}(1, s) \mu_{q}(1, t)$, and hence applying GAD along with replication and scale invariance, we obtain

$$
I(1, s, t, s t)=\left[\bar{f}\left(1 / \mu_{q}(1, t)\right)+\bar{f}\left(t / \mu_{q}(1, t)\right)\right] I(1, s)+I(1, t) .
$$

Similarly,

$$
I(1, t, s, t s)=\left[\bar{f}\left(1 / \mu_{q}(1, s)\right)+\bar{f}\left(s / \mu_{q}(1, s)\right)\right] I(1, t)+I(1, s),
$$

and hence by symmetry of $I$,

\footnotetext{
${ }^{9}$ Note that if $(r, a, b)$ and $(s, b, d)$ are both in the domain of $f$, then $(r s, a, d)$ is also. For example, if $q>0$, then $2 \leq a<b$ and $r \in\left(0,(b / a)^{1 / q}\right)$ together with $2 \leq b<d$ and $s \in\left(0,(d / b)^{1 / q}\right)$ imply $2 \leq a<d$ and $r s \in\left(0,(d / a)^{1 / q}\right)$.
} 


$$
\begin{aligned}
{\left[\bar{f}\left(1 / \mu_{q}(1, t)\right)\right.} & \left.+\bar{f}\left(t / \mu_{q}(1, t)\right)-1\right] I(1, s) \\
& =\left[\bar{f}\left(1 / \mu_{q}(1, s)\right)+\bar{f}\left(s / \mu_{q}(1, s)\right)-1\right] I(1, t),
\end{aligned}
$$

which holds for all $s, t>0$. Now fix $t>1$ and define $\beta=\left[\bar{f}\left(1 / \mu_{q}(1, t)\right)+\right.$ $\left.\bar{f}\left(t / \mu_{q}(1, t)\right)-1\right] / I(1, t)$, where $I(1, t) \neq 0$ follows from normalization. Then

$$
\beta I(1, s)=\bar{f}\left(1 / \mu_{q}(1, s)\right)+\bar{f}\left(s / \mu_{q}(1, s)\right)-1,
$$

which in turn implies

$$
\beta I(1, s)+1 \geq \bar{f}\left(1 / \mu_{q}(1, s)\right)
$$

for all $s>0$, since the weights (and hence $\bar{f}$ ) are nonnegative by assumption. The two-income transfer principle and scale invariance ensure that $I(1, s)$ is bounded in $s$ on, say, the interval [1/2,2]. Therefore, defining $r_{1}=1 / \mu_{q}(1,2)<1$ and $r_{2}=1 / \mu_{q}(1,1 / 2)<1$, we conclude that $\bar{f}(r)$ and hence $f(r, a, b)$ for $2 \leq a<b$ is bounded on an interval $\left[r_{1}, r_{2}\right]$ that includes 1 in its interior.

Now (5) is a conditional Pexider-type equation (see Aczél and Dombres, 1989), whose general "bounded on an interval" solution is of the form ${ }^{10}$

$$
f(r, a, b)=\phi(a, b) r^{c}
$$

where $c \in R$ is arbitrary and $\phi(a, b)=f(1, a, b)$. The next step is to solve for the functional form of $\phi$. Substituting $r=s=1$ in (5) yields the functional equation

$$
\phi(a, b) \phi(b, d)=\phi(a, d)
$$

which must hold for all natural numbers $a, b, d$ with $2 \leq a<b<d$. If we select in particular $d=a b$, then (7) reduces to

$$
\phi(a, b) \phi(b, a b)=\phi(a, a b)
$$

for natural numbers $a, b$ satisfying $2 \leq a<b$. It can be shown that $\phi(k, \ell)=k / \ell$ for all natural numbers $k, \ell \geq 2$. Indeed, let $x$ be any distribution satisfying $n(x)=k$ and $I(x) \neq 0$, and let $y$ be an $\ell$-replication of $x$. Then by replication invariance, GAD and normalization, we obtain $I(x)=I(y)=\ell \phi(k, k \ell) I(x)$, and consequently $\phi(k, k \ell)=1 / \ell$ for natural numbers $k, \ell \geq 2$. Applying this twice to (8) yields $\phi(a, b)=a / b$ and hence from (6) we obtain

$$
f(r, a, b)=(a / b) r^{c}
$$

for some $c \in R$, given $2 \leq a<b$. Consequently, by (4) we conclude that for any relative inequality measure satisfying GAD for $q \in R$, the weights must have the form

$$
w^{j}\left(\boldsymbol{\mu}_{\mathbf{q}}, \mathbf{n}\right)=\left(n^{j} / n\right)\left(\mu_{q}^{j} / \mu_{q}\right)^{c}
$$

for some parameter $c \in R$, for any $m \geq 2$ and all $j$ satisfying $n^{j} \geq 2$. This completes the proof.

\footnotetext{
${ }^{10}$ It can be shown that $\lambda(s)=\ln \left[f\left(e^{s}, a, d\right)\right]-\ln [f(1, a, d)]$ satisfies $\lambda(s+t)=\lambda(s)+\lambda(t)$ for all pairs $(s, t) \in(-\infty, \ln (b / a)) \times(-\infty, \ln (d / b))$. The domain contains $(0,0)$ in its interior, and therefore, as Aczel and Dombres (1989) demonstrate, the general bounded in an interval solution of the above equation is $\lambda(s)=c s$ with arbitrary $c \in R$.
} 
Proposition 1 shows that any measure that satisfies GAD must actually satisfy a stronger decomposition formula in which the weights take on the form (1). This includes the "population-share" weighting structure of the $I_{q}$ measures and the "income share" weighting of the first Theil measure. The weighting structure closely resembles that of the generalized entropy measures $\left(n^{j} / n\right)\left(\mu^{j} / \mu\right)^{c}$, but with a $q$-order mean used in place of the arithmetic mean. Thus the weights depend on the population share of the subgroup as well as its relative level of prosperity as measured by a generalized mean.

The results given in Proposition 1 will now be used to characterize the entire class of measures satisfying GAD. Consider the following two-parameter family of inequality measures:

$$
I_{c q}(x)= \begin{cases}\frac{1}{c(c-q)}\left[\left(\frac{\mu_{c}(x)}{\mu_{q}(x)}\right)^{c}-1\right], & c \neq 0, q \neq c \\ \frac{1}{q} \frac{1}{n} \sum_{i=1}^{n}\left(\frac{x_{i}}{\mu_{q}(x)}\right)^{q} \ln \left(\frac{x_{i}}{\mu_{q}(x)}\right), & c \neq 0, q=c \\ \frac{1}{q} \ln \left(\frac{\mu_{q}(x)}{g(x)}\right) & c=0, q \neq c \\ \frac{1}{2} V_{L}(x), & c=0, q=c\end{cases}
$$

It can be shown that $I_{c q}$ satisfies all five of the basic properties for inequality measures described above. For example, in the generic case of $c \neq 0$ and $q \neq c$, the measure is based on the ratio of two generalized means, $\mu_{c}$ and $\mu_{q}$, and consequently symmetry, replication invariance and scale invariance hold. As for normalization, it is clear that $\mu_{q}(x)$ is constant in $q$ whenever $x$ is completely equal, and hence $I_{c q}(x)=0$ in this case; while a result in Hardy, Littlewood and Polya (1952, p. 26) ensures that $\mu_{q}(x)$ is strictly increasing in $q$ when $x$ is not completely equal, and consequently, $I_{c q}(x)>0$ for this case. The twoperson transfer principle follows from a well-known result on strictly Schurconvex functions (see for example Berge, 1963, p. 221) applied to two-person distributions. Similar arguments may be made for the remaining values of the parameters.

All of the previously defined measures are represented in this class for appropriate values of the parameters. The generalized entropy measures are obtained when $q=1$, with limiting measures $I_{11}=T_{1}$ and $I_{01}=T_{2}$. The $I_{q}$ measures arise when $c=0$, with limiting measure $I_{00}=V_{L} / 2$. As noted above, all of these examples have a general additive decomposition. In fact, this is true for all $I_{c q}$ measures and moreover, as the next result shows, they are essentially the only measures satisfying GAD.

Proposition 2. I is a relative inequality measure satisfying GAD if and only if I is a positive multiple of $I_{c q}$ for some $c, q \in R$.

Proof. One can easily verify that $I_{c q}$ has a general additive decomposition of the form 


$$
I_{c q}\left(x^{1}, \ldots, x^{m}\right)=\sum_{j=1}^{m} \frac{n^{j}}{n}\left(\frac{\mu_{q}^{j}}{\mu_{q}}\right)^{c} I_{c q}\left(x^{j}\right)+I_{c q}\left(\left(\mu_{q}\left(x^{1}\right) u_{n^{1}}, \ldots, \mu_{q}\left(x^{m}\right) u_{n^{m}}\right),\right.
$$

for each collection $x^{1}, \ldots, x^{m} \in D$. In addition, $I_{c q}$ clearly satisfies symmetry, replication invariance, scale invariance and the transfer principle for two incomes, and so necessity is assured. For sufficiency, suppose that $I$ is a relative inequality measure satisfying GAD for some $q \in R$. We will first show that for any distribution $x$ with $n(x)=2$, we have $I(x)=\alpha I_{c q}(x)$ for some $\alpha>0$ and some $c \in R$. There are two cases: $q \neq 0$ and $q=0$.

In the case of $q \neq 0$, let $J: D \rightarrow R$ be defined by $J(x)=I\left((x)^{1 / q}\right)$ for $x \in D$. It is straightforward to verify that $J$ satisfies all the properties required of a relative inequality measure, as well as GAD for $q=1$. Now consider the distribution $(p, r, s, t) \in D$ with $p+r+s+t=1$. By GAD and Proposition 1, there is a $c \in R$ such that

$$
\begin{aligned}
J(p, r, s, t)= & 2^{c-1}(p+r)^{c} J(p, r)+2^{c-1}(s+t)^{c} J(s, t) \\
& +J\left(\mu(p, r) u_{2}, \mu(s, t) u_{2}\right) .
\end{aligned}
$$

Let $f:(0,1) \rightarrow R_{+}$be defined by $f(u)=J(u, 1-u)$. Then using the properties of $J$, we may rewrite (9) as

$J(p, r, s, t)=2^{c-1}(p+r)^{c} f\left(\frac{p}{p+r}\right)+2^{c-1}(1-p-r)^{c} f\left(\frac{s}{1-p-r}\right)+f(p+r)$.

Similarly,

$J(p, s, r, t)=2^{c-1}(p+s)^{c} f\left(\frac{p}{p+s}\right)+2^{c-1}(1-p-s)^{c} f\left(\frac{r}{1-p-s}\right)+f(p+s)$,

and therefore, by symmetry of $J$, we have the following functional equation for $f$ :

$$
\begin{aligned}
& f(p+r)+2^{c-1}(1-p-r)^{c} f\left(\frac{s}{1-p-r}\right)+2^{c-1}(p+r)^{c} f\left(\frac{p}{p+r}\right) \\
& \quad=f(p+s)+2^{c-1}(p+s)^{c} f\left(\frac{p}{p+s}\right)+2^{c-1}(1-p-s)^{c} f\left(\frac{r}{1-p-s}\right),
\end{aligned}
$$

which holds for all $p, r, s \in(0,1)$ with $p+r+s<1$. The next step is to characterize the function $f$.

Before finding the solution to (10), we show that $f$ is continuously differentiable of all orders, or $f \in C^{\infty}(0,1)$. Set $p=r=v / 2$ in (10) to obtain

$$
\begin{aligned}
f(v) & +2^{c-1}(1-v)^{c} f\left(\frac{s}{1-v}\right) \\
& =f\left(s+\frac{v}{2}\right)+2^{c-1}\left(s+\frac{v}{2}\right)^{c} f\left(\frac{v}{v+2 s}\right) \\
& +2^{c-1}\left(1-s-\frac{v}{2}\right)^{c} f\left(\frac{v}{2-v-2 s}\right),
\end{aligned}
$$


where normalization of $J$ (i.e., $f(1 / 2)=0$ ) has been invoked. The two-person transfer principle implies that $f$ is monotonic on $(0,1 / 2]$ and on $[1 / 2,1)$. Therefore $f$ is Lebesgue integrable on every interval $\left[r_{1}, r_{2}\right] \subset(0,1)$. For any $v \in(0,1)$, choose $r_{1}, r_{2}$ so that $0<r_{1}<r_{2}<1-v$ and integrate (11) on $\left[r_{1}, r_{2}\right]$ with respect to $s$ to obtain

$$
\begin{aligned}
& \left(r_{2}-r_{1}\right) f(v)=-2^{c-1} \int_{r_{1}}^{r_{2}}(1-v)^{c} f\left(\frac{s}{1-v}\right) d s+\int_{r_{1}}^{r_{2}} f\left(s+\frac{v}{2}\right) d s \\
& +2^{c-1} \int_{r_{1}}^{r_{2}}\left(s+\frac{v}{2}\right)^{c} f\left(\frac{v}{v+2 s}\right) d s+2^{c-1} \int_{r_{1}}^{r_{2}}\left(1-s-\frac{v}{2}\right)^{c} f\left(\frac{v}{2-v-2 s}\right) d s
\end{aligned}
$$

Changing variables in each of the integrals in (12) yields

$$
\begin{aligned}
& \left(r_{1}-r_{2}\right) f(v)=-2^{c-1}(1-v)^{c+1} \int_{r_{1} /(1-v)}^{r_{2} /(1-v)} f(u) d u+\int_{r_{1}+v / 2}^{r_{2}+v / 2} f(u) d u \\
& +(1 / 4) v^{c+1} \int_{v /\left(v+2 r_{2}\right)}^{v /\left(v+2 r_{1}\right)} f(u) / u^{c+2} d u+(1 / 4) v^{c+1} \int_{v /\left(2-v-2 r_{1}\right)}^{v /\left(2-v-2 r_{2}\right)} f(u) / u^{c+2} d u
\end{aligned}
$$

where $0<r_{1}<r_{2}<1-v$ ensures that $0<r_{1} /(1-v)<r_{2} /(1-v)<$ $1,0<r_{1}+v / 2<r_{2}+v / 2<1,0<v /\left(v+2 r_{2}\right)<v /\left(v+2 r_{1}\right)<1$, and $0<v /\left(2-v-2 r_{1}\right)<v /\left(2-v-2 r_{2}\right)<1$, so that all integrals in (13) are well-defined. The right side of (13) is clearly continuous in $v \in(0,1)$, and so $f$ must be continuous as well. But then the right side is differentiable in $v \in(0,1)$, and hence $f$ is differentiable. Iteration of this argument gives $f \in C^{\infty}(0,1)$, as we set out to show.

Now returning to the functional equation (10), let us differentiate twice once with respect to $r$ and then once with respect to $s-$ to obtain

$$
\begin{aligned}
& (1-p-s)^{c-2}\left[(1-c) f^{\prime}\left(\frac{r}{1-p-s}\right)+\left(\frac{r}{1-p-s}\right) f^{\prime \prime}\left(\frac{r}{1-p-s}\right)\right] \\
& =(1-p-r)^{c-2}\left[(1-c) f^{\prime}\left(\frac{s}{1-p-r}\right)\right. \\
& \left.\quad+\left(\frac{s}{1-p-r}\right) f^{\prime \prime}\left(\frac{s}{1-p-r}\right)\right] .
\end{aligned}
$$

Changing variables $u=r /(1-p-s)$ and $v=s /(1-p-r)$ transforms (14) into

$$
(1-u)^{2-c}\left[(1-c) f^{\prime}(u)+u f^{\prime \prime}(u)\right]=(1-v)^{2-c}\left[(1-c) f^{\prime}(v)+v f^{\prime \prime}(v)\right],
$$

an equation that must hold for all $u, v \in(0,1)$. Therefore

$$
(1-u)^{2-c}\left[(1-c) f^{\prime}(u)+u f^{\prime \prime}(u)\right]=\beta,
$$

for all $u \in(0,1)$, where $\beta \in R$ is a constant. This is a second-order linear differential equation whose solution on $(0,1)$ satisfying $f(u)>f(1 / 2)=0$ for all $u \in(0,1)$ with $u \neq 1 / 2$ is of the form 


$$
f(u)= \begin{cases}\frac{\alpha^{\prime}}{c(c-1)}\left[(1-u)^{c}+u^{c}-2^{1-c}\right] & c \neq 0,1 \\ -\alpha^{\prime}[(1 / 2) \ln (2 u)+(1 / 2) \ln (2(1-u))] & c=0 \\ \alpha^{\prime}[u \ln (2 u)+(1-u) \ln (2(1-u))] & c=1\end{cases}
$$

where $\alpha^{\prime}>0$ is a constant. Now recalling that $J(x)=f\left(x_{1} /(2 \mu(x))\right)$ for $x=$ $\left(x_{1}, x_{2}\right) \in R_{++}^{2}$, it follows that $J$ must have the form

$$
J(x)= \begin{cases}\frac{\alpha}{c(c-1)}\left[\left(\frac{x_{1}}{\mu(x)}\right)^{c}+\left(\frac{x_{2}}{\mu(x)}\right)^{c}-2\right], & c \neq 0,1 \\ -\frac{\alpha}{2}\left[\ln \left(\frac{x_{1}}{\mu(x)}\right)+\ln \left(\frac{x_{2}}{\mu(x)}\right)\right], & c=0 \\ \frac{\alpha}{2}\left[\frac{x_{1}}{\mu(x)} \ln \left(\frac{x_{1}}{\mu(x)}\right)+\frac{x_{2}}{\mu(x)} \ln \left(\frac{x_{2}}{\mu(x)}\right)\right], & c=1\end{cases}
$$

for $x=\left(x_{1}, x_{2}\right) \in R_{++}^{2}$, where $\alpha>0$ is a constant. Finally making use of the fact that $I(x)=J\left((x)^{q}\right)$, we conclude that in the case where $q \neq 0$,

$$
I(x)=\alpha I_{c q}(x)
$$

for $x \in R_{++}^{2}$, for some constants $\alpha>0$ and $c \in R$.

Now for the case of $q=0$, define $X=\cup_{n \geq 1} R^{n}$ and let $A: X \rightarrow R$ be defined by $A(x)=I\left(e^{(x)}\right)$, where we recall that $e^{(x)}=\left(e^{x_{1}}, \ldots, e^{x_{n(x)}}\right)$. It is easy to verify that $A$ satisfies symmetry, replication invariance, normalization, and the two-income transfer principle on the broadened domain $X$. However, $A$ is translation invariant rather than scale invariant, so that $A\left(x+\alpha u_{n(x)}\right)=A(x)$ for any $\alpha \in R$ and $x \in X$. Nevertheless, the proof in this case is quite similar to the $q \neq 0$ proof, with $A$ now playing the role of $J$.

Consider the vector $(p, r, s, t) \in X$ with $p+r+s+t=0$. Applying Proposition 1 and GAD to $I$ yields

$$
\begin{aligned}
A(p, r, s, t) & =1 / 2 e^{c \mu(p, r)} A(p, r)+1 / 2 e^{c \mu(s, t)} A(s, t)+A(\mu(p, r), \mu(s, t)) \\
& =1 / 2 e^{c \mu(p, r)} A(p-\mu(p, r), r-\mu(p, r)) \\
& +1 / 2 e^{c \mu(s, t)} A(s-\mu(s, t), t-\mu(s, t))+A(\mu(p, r), \mu(s, t))
\end{aligned}
$$

where replication invariance of $I$ and translation invariance of $A$ have been invoked. Now defining the function $f: R \rightarrow R$ by $f(u)=A(u,-u)$ for all $u \in R$, we obtain

$$
A(p, r, s, t)=1 / 2 e^{c \mu(p, r)} f(p-\mu(p, r))+1 / 2 e^{-c \mu(p, r)} f(s+\mu(p, r))+f(\mu(p, r)) .
$$

A similar expression can be derived for $A(p, s, r, t)$, so that by symmetry of $A$ we have the functional equation

$$
\begin{aligned}
& 1 / 2 e^{c \mu(p, r)} f(p-\mu(p, r))+1 / 2 e^{-c \mu(p, r)} f(s+\mu(p, r))+f(\mu(p, r)) \\
& =1 / 2 e^{c \mu(p, s)} f(p-\mu(p, s))+1 / 2 e^{-c \mu(p, s)} f(r+\mu(p, s))+f(\mu(p, s)),
\end{aligned}
$$


which holds for all $p, r, s \in R$. Before solving (16) we first establish regularity properties for the function $f$.

Set $p=r=v$ in (16) to obtain

$$
\begin{aligned}
f(v)= & -1 / 2 e^{-c v} f(s+v)+1 / 2 e^{c\left(\frac{v+s}{2}\right)} f\left(\frac{v-s}{2}\right) \\
& +1 / 2 e^{-c\left(\frac{v+s}{2}\right)} f\left(\frac{3 v+s}{2}\right)+f\left(\frac{v+s}{2}\right),
\end{aligned}
$$

where $f(0)=0$ has been invoked. The two-person transfer principle implies that $f$ is monotonic on each of the intervals $(-\infty, 0]$ and $[0, \infty)$, and so $f$ is Lebesgue integrable on every interval in $R$. Integrating (17) on $\left[r_{1}, r_{2}\right] \subset R$ with respect to $s$ yields

$$
\begin{aligned}
& \left(r_{2}-r_{1}\right) f(v)=-1 / 2 \int_{r_{1}}^{r_{2}} e^{-c v} f(s+v) d s \\
& \quad+1 / 2 \int_{r_{1}}^{r_{2}} e^{c\left(\frac{v+s}{2}\right)} f\left(\frac{v-s}{2}\right) d s+1 / 2 \int_{r_{1}}^{r_{2}} e^{-c\left(\frac{v+s}{2}\right)} f\left(\frac{3 v+s}{2}\right) d s \\
& \quad+\int_{r_{1}}^{r_{2}} f\left(\frac{v+s}{2}\right) d s
\end{aligned}
$$

After several changes of variables, we obtain

$$
\begin{aligned}
& \left(r_{2}-r_{1}\right) f(v)=-1 / 2 e^{-c v} \int_{r_{1}+v}^{r_{2}+v} f(u) d u+e^{c v} \int_{\left(v-r_{2}\right) / 2}^{\left(v-r_{1}\right) / 2} e^{-c u} f(u) d u \\
& +e^{c v} \int_{\left(3 v+r_{1}\right) / 2}^{\left(3 v+r_{2}\right) / 2} e^{-c u} f(u) d u+2 \int_{\left(v+r_{1}\right) / 2}^{\left(v+r_{2}\right) / 2} f(u) d u .
\end{aligned}
$$

As before, since the right side is continuous in $v \in R$, we find that $f$ is continuous, and so forth, so that $f \in C^{\infty}(0,1)$.

To solve (16), set $p=0$ and differentiate twice - once with respect to $r$ and a second time with respect to $s$ - to obtain

$$
\begin{aligned}
& 1 / 2 e^{-c r / 2} f^{\prime \prime}\left(s+\frac{r}{2}\right)-c / 2 e^{-c r / 2} f^{\prime}\left(s+\frac{r}{2}\right) \\
& =1 / 2 e^{-c s / 2} f^{\prime \prime}\left(r+\frac{s}{2}\right)-c / 2 e^{-c s / 2} f^{\prime}\left(r+\frac{s}{2}\right)
\end{aligned}
$$

Setting $r=-2 u / 3$ and $s=4 u / 3$ in (18) yields

$$
f^{\prime \prime}(u)-c f^{\prime}(u)=\beta e^{-c u},
$$

where $\beta$ is a constant. For $c \neq 0$, the general solution of (19) satisfying $f(u)>$ $f(0)=0$ for all $u \in R$ with $u \neq 0$ has the form

$$
f(u)=\alpha\left[\mu\left(e^{c u}, e^{-c u}\right)-1\right],
$$

where $\alpha>0$ is a constant; while for $c=0$ the solution is 


$$
f(u)=(\alpha / 2) u^{2},
$$

for some constant $\alpha>0$. In view of $A(x)=f\left(x_{1}-\mu(x)\right)$ for $x=\left(x_{1}, x_{2}\right) \in R^{2}$, it follows that $A$ must have the form

$$
A(x)= \begin{cases}\frac{\alpha \mu\left(e^{c x}\right)}{e^{c \mu(x)}}-1 & c \neq 0 \\ \frac{\alpha}{2} V(x) & c=0\end{cases}
$$

for $x \in R^{2}$, where $\alpha>0$ is a constant. Finally, making use of $I(x)=A(\ln (x))$ for $x \in R_{++}^{2}$, we obtain $I(x)=\alpha I_{c q}(x)$ for some constants $\alpha>0$ and $c \in R$, for the remaining case of $q=0$.

Having established (15) for all $q \in R$ for distributions of size 2, we now show that it holds for distributions of size $2^{k}$ for any natural number $k$. Suppose that $I(x)=\alpha I_{c q}(x)$ for all distributions of sizes $2^{j}$ for $j=1, \ldots, k-1$, where $k \geq 2$. Then given any $x \in D$ with $n(x)=2^{k}$, split $x$ into $y \in D$ and $z \in D$ with $n(y)=n(z)=2^{k-1}$. By the induction hypothesis, $I(y)=\alpha I_{c q}(y)$ and $I(z)=\alpha I_{c q}(z)$, and indeed $I(\mu(y), \mu(z))=\alpha I_{c q}(\mu(y), \mu(z))$. GAD and Proposition 1 , along with replication invariance, imply that

$$
\begin{aligned}
I(x) & \left.=1 / 2\left(\frac{\mu_{q}(y)}{\mu_{q}(x)}\right)^{c} I(y)+1 / 2\left(\frac{\mu_{q}(z)}{\mu_{q}(x)}\right)^{c} I(z)+I\left(\mu_{q}(y), \mu_{q}(z)\right)\right] \\
& =\alpha\left[1 / 2\left(\frac{\mu_{q}(y)}{\mu_{q}(x)}\right)^{c} I_{c q}(y)+1 / 2\left(\frac{\mu_{q}(z)}{\mu_{q}(x)}\right)^{c} I_{c q}(z)+I_{c q}\left(\mu_{q}(y), \mu_{q}(z)\right)\right] \\
(20) & =\alpha I_{c q}(x),
\end{aligned}
$$

by the decomposition property satisfied by $I_{c q}$. Consequentially, (15) holds for all distributions of size $2^{k}$ for arbitrary $k$.

To complete the proof, let $x \in D$ be any given distribution, and let $y$ be a completely equal distribution satisfying $\mu_{q}(x)=\mu_{q}(y)$ and $n(x)+n(y)=2^{k}$ for some $k$. Then by GAD, Proposition 1 and normalization, $I(x, y)=\frac{n(x)}{n(x, y)} I(x)$, while $I_{c q}(x, y)=\frac{n(x)}{n(x, y)} I_{c q}(x)$ by the definition of $I_{c q}$. Consequentially, (15) holds for all $x \in D$, which completes the proof.

Our method of proof follows the standard approach to characterizing inequality measures using functional equation methods: We derive a functional equation (i.e., (10) and (16) above) from the properties placed on the inequality measure $I$, and then find its solution, which in turn is used to obtain the functional form of $I$. However, there are two aspects of the proof that should be mentioned. First, it does not require any regularity assumptions (such as continuity of $I$ ) to solve the functional equation, but rather derives the needed smoothness from basic principles. ${ }^{11}$ Second, the proof is entirely self-contained. Once we ensure

\footnotetext{
11 This is also the approach of Foster (1983) and Gehrig (1983). Of course, if continuity were assumed from the outset, the argument would be correspondingly shortened. For example, the results of Shorrocks $(1980,1984)$ can be applied directly in the case $q \neq 0$ to obtain the functional form for $I$ in the presence of continuity. Specifically, the function $J(x)=I\left((x)^{1 / q}\right)$ can be shown to satisfy the $A D$ axiom as well as the other properties assumed by Shorrocks and hence $I$ must be a generalized entropy measure applied to $(x)^{q}$.
} 
smoothness of the function in question, we simply differentiate the functional equation to obtain a differential equation that can be solved by standard methods.

\section{Discussion}

Proposition 2 identifies the two-parameter family $I_{c q}$ as the collection of all measures satisfying GAD. One way of viewing this result is to fix $q$ and note that there is a single-parameter class of measures with a general additive decomposition based on that specific $\mu_{q}$. For example, when $q=1$ we obtain the well-known generalized entropy measures, which utilize $\mu$ in their decompositions. Another example of interest is $q=0$, which generates the class

$$
I_{c 0}(x)= \begin{cases}\frac{1}{c^{2}}\left[\left(\frac{\mu_{c}(x)}{g(x)}\right)^{c}-1\right] & c \neq 0 \\ \frac{1}{2} V_{L}(x) & c=0\end{cases}
$$

This extends the variance of logarithms to an entire family of measures that are decomposable relative to the geometric mean.

Alternatively, one could fix $c$ and allow $q$ to vary to obtain a single-parameter family of measures, one for each $q$. A typical example of this is the $I_{q}$ family discussed earlier, which arises when $c=0$. A second example of this approach is the new family of measures

$$
I_{1 q}(x)= \begin{cases}\frac{1}{1-q}\left[\frac{\mu(x)-\mu_{q}(x)}{\mu_{q}(x)}\right] & q \neq 1 \\ T_{1} & q=0\end{cases}
$$

obtained by setting $c=1$. We may recall that Atkinson's (1970) parametric family takes on the form $A_{q}=\left(\mu-\mu_{q}\right) / \mu$ for some $q<1$, where $\mu_{q}=\mu_{q}(x)$ is interpreted as the welfare level of the actual distribution $x$ and $\mu=\mu(x)$ is a proxy for the maximum possible welfare for a given total income. ${ }^{12}$ Clearly, $I_{c q}$ has a form reminiscent of Atkinson's measures, but using the actual welfare level as the base rather than the maximum welfare level. While Atkinson's measures evaluate the percentage welfare loss from inequality, $I_{1 q}$ can be interpreted as the percentage welfare gain in moving from the actual distribution to complete equality.

One interesting characteristic of the $I_{c q}$ measures is that the inequality value $I_{c q}(x)$ varies continuously as a function of the parameters at each $x \in D$. The verification of this fact is, of course, trivial for the generic case of $c \neq 0$ and

\footnotetext{
12 In Atkinson's framework, the generalized mean $\mu_{q}$ is the "equally distributed equivalent income" function, a special linearly homogeneous cardinalization of the welfare function. The maximum welfare for a given total income is $\mu_{q}\left(x^{*}\right)$, where $x^{*}$ is the completely equal distribution with the same mean as $x$. But since $\mu(x)=\mu\left(x^{*}\right)=\mu_{q}\left(x^{*}\right)$, we see that $\mu(x)$ is the maximum possible welfare.
} 
$q \neq c$; the remaining cases require applications of l'Hopital's rule. Note that the limiting classes $\left\{I_{c q}: q=c\right\}$ and $\left\{I_{c q}: c=0\right\}$ are generalizations of the first and second Theil measures, respectively. Moreover, since the main generalized entropy formula may be rewritten as $I_{c}(x)=\left[\left(\mu_{c}(x) / \mu(x)\right)^{c}-1\right] /[c(c-1)]$, it is clear that $I_{c q}$ is a straightforward extension in this case as well. It is also interesting to note that (half) the variance of logarithms is located at the intersection of the two Theil subclasses, and that each of the other three formulas for $I_{c q}$ (the generic and the two Theil formulas) converges to $V_{L} / 2$ as the parameters tend to 0 . This intriguing relationship between the generalized entropy class (including the two Theil measures) on one hand, and the variance of logarithms, on the other, has apparently escaped notice.

Now what properties beyond the ones used to characterize $I_{c q}$ are satisfied by the measures? First, although no assumption of continuity was made in the above propositions, all of the resulting measures are continuous in $x$. This follows immediately from the definition of $I_{c q}$, recalling that continuity is only required over each $n$-person slice of the domain $D$. Second, while we have assumed the two-income transfer principle in our characterization, it is clear that this does not necessarily yield the full transfer principle - even for measures satisfying GAD. To see this, select any distribution $x$ partitioned into $x^{1}$ with two incomes and $x^{2}$ with one or more. A regressive transfer in the first group clearly raises its inequality level and leaves the second group's level unchanged. Now, if the within-group weights and the between-group smoothed distribution were unaffected, then GAD would ensure that overall inequality would rise as well. But, in general, the subgroup data $\left(\boldsymbol{\mu}_{\mathbf{q}}, \mathbf{n}\right)$ will be altered as a result of the transfer. This in turn will change the subgroup weights and the smoothed distribution, allowing the possibility that overall inequality falls. ${ }^{13}$ In the special case of $q=1$, which corresponds to the usual additive decomposability, the subgroup data is unaffected by the (mean-preserving) transfer, and the two transfer axioms are indeed equivalent. Thus the generalized entropy measures are counted among the measures that satisfy the transfer principle. But this route to the transfer principle does not generally apply for the other $I_{c q}$ measures.

Nonetheless, it turns out that a significant subclass does, in fact, satisfy the stronger axiom. By a well-known result (see, for example, Berge, 1963, p. 221), $I_{c q}$ satisfies the transfer principle if for any $x \in D$ we have

$$
\frac{\partial I_{c q}(x)}{\partial x_{i}}>\frac{\partial I_{c q}(x)}{\partial x_{j}}
$$

for all $i, j$ with $x_{i}>x_{j}$. Once again, let us focus on the generic case of $c \neq 0$ and $q \neq c$. After some algebra we obtain

$$
\frac{\partial I_{c q}(x)}{\partial x_{i}}=\frac{1}{n(x)(c-q)} \frac{1}{\left[\mu_{q}(x)\right]^{c}}\left(x_{i}^{c-1}-\frac{\left[\mu_{c}(x)\right]^{c}}{\left[\mu_{c}(x)\right]^{q}} x_{i}^{q-1}\right)
$$

Consequently, (20) holds whenever

13 This is closely related to the notion of "subgroup consistency", on which see Shorrocks (1988) and Foster and Sen (1997). 


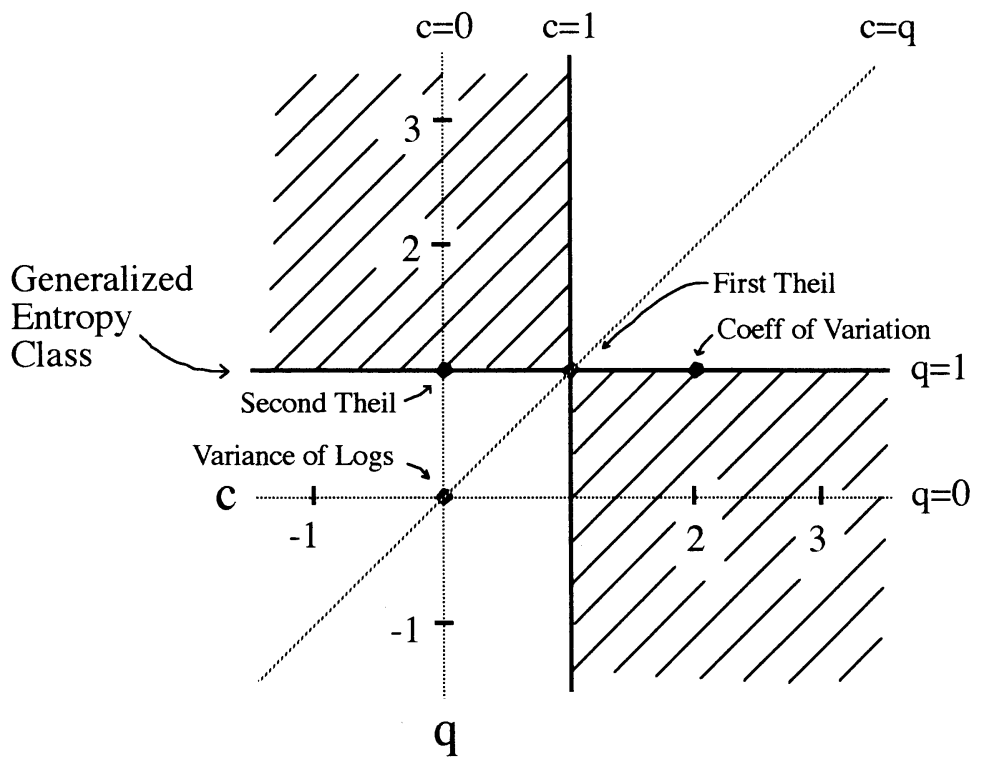

Figure 1. $I_{c q}$ measures and the transfer principle

$$
\frac{1}{c-q}\left[\left(x_{i}^{c-1}-x_{j}^{c-1}\right)-\gamma(x)\left(x_{i}^{q-1}-x_{j}^{q-1}\right)\right]>0
$$

where $\gamma(x)=\left[\mu_{c}(x)\right]^{c} /\left[\mu_{q}(x)\right]^{q}$. Obviously if $c \geq 1$ and $q \leq 1$, or if $c \leq 1$ and $q \geq 1$, the transfer principle holds. On the other hand, if $c$ and $q$ are both positive or both negative, then it is possible to construct a distribution $x$ for which (20) is violated. Combining these conclusions with the analogous calculations for the limiting cases yields the following result: $I_{c q}$ satisfies the transfer principle if and only if either (i) $c \geq 1$ and $q \leq 1$ or (ii) $c \leq 1$ and $q \geq 1$.

The measures satisfying the transfer principle are represented in Fig. 1 as the shaded regions defined by the axes centered at $(c, q)=(1,1)$. The generalized entropy measures $I_{c 1}$ are found along the horizontal axis while the family $I_{1 q}$, which resembles the Atkinson family, lies on the vertical axis. These two families are the only single-parameter classes lying entirely within the shaded regions. All others, including the $I_{0 q}$ and $I_{c 0}$ families discussed above are bisected into ranges that satisfy and violate the property. As for specific measures, $I_{00}=V_{L} / 2$ lies outside the shaded area, while the two Theil measures and the (squared) coefficient of variation are part of that set. ${ }^{14}$

The shaded region in Fig. 1 is quite clearly symmetric with respect to the 45-degree line defined by $c=q$. This suggests that a relationship might exist between $I_{c q}$ and $I_{q c}$. From the definition of $I_{c q}$ it follows that

\footnotetext{
${ }^{14}$ See Foster and Ok (1999) on the failure of the variance of logarithms to satisfy the transfer principle. Note that while a large subset of the $I_{c q}$ measures violate the transfer principle, all of them (including $V_{L}$ ) satisfy the two-person version as shown in Proposition 2.
} 


$$
(c-q)\left[I_{c q}(x)-I_{c q}(y)\right]>0 \text { if and only if } \frac{\mu_{c}(x)}{\mu_{q}(x)}>\frac{\mu_{c}(y)}{\mu_{q}(y)} .
$$

Similarly, after some algebra, we obtain

$$
(c-q)\left[I_{q c}(x)-I_{q c}(y)\right]>0 \text { if and only if } \frac{\mu_{c}(x)}{\mu_{q}(x)}>\frac{\mu_{c}(y)}{\mu_{q}(y)} .
$$

Combining these two observations, then leads to the result: $I_{q c}$ is a positive monotonic transformation of $I_{c q}$ for $c \neq q .{ }^{15}$ Consequently, every measure satisfying GAD for $\mu_{q}$ has a monotonic transformation that satisfies GAD for some other $\mu_{c}$. In particular, if $I_{c q}$ satisfies the transfer axiom, then so must its "dual" measure $I_{q c}$, which in turn explains the symmetry of the shaded region. This also implies that every "Atkinson-related" measure $I_{1 q}$ is a positive monotonic transformation of some generalized entropy measure $I_{c 1}$, and vice versa, so that the class $I_{1 q}$ may be viewed as a "dual" generalized entropy class whose members are decomposable with respect to generalized means. ${ }^{16}$ Interestingly, the generalized entropy class and its dual comprise the boundary of the set of measures satisfying the transfer principle, and in this sense "just satisfy" this central axiom.

We conclude this section by noting an additional link between $I_{c q}$ and the generalized entropy measures. Select any nonzero $q$, construct the distribution $(x)^{q}$ by raising each entry of $x$ to the $q$ th power, and let $c^{\prime}=c / q$. It can be verified that

$$
I_{c q}(x)=\left(1 / q^{2}\right) I_{c^{\prime}}\left((x)^{q}\right)
$$

where $I_{c^{\prime}}$ is the generalized entropy measure with parameter $c^{\prime}$. In other words, to find the inequality level as measured by $I_{c q}$, transform incomes and apply the generalized entropy measure whose parameter is the ratio $c / q$. So, for example, the class $\left\{I_{c q}: q=1\right\}$ is the generalized entropy class; the class $\left\{I_{c q}: c=0\right\}$ is obtained by applying the second Theil measure to $(x)^{q}$; and $\left\{I_{c q}: q=c\right\}$ arises when the first Theil measure is appled to $(x)^{q}$. If we restrict consideration to $0<q<1$, then it is possible to interpret $x_{i}^{q}$ as a strictly concave utility function for person $i$. In this case, $I_{c q}(x)$ has the intuitive interpretation as the inequality in the distribution of utility as measured by a generalized entropy measure. ${ }^{17}$

\footnotetext{
15 Alternatively, $I_{q c}=\left[\left(1+c(c-q) I_{c q}\right)^{-q / c}-1\right] /[q(q-c)]$, which can be shown to be increasing in $I_{c q}$. Notice that while $I_{c q}$ and $I_{q c}$ rank distributions in the same way, the respective decompositions are different.

16 Thus every member $A_{q}$ of Atkinson's single parameter class is a monotonic transformation of some $I_{1 q}$ measure. Given the nice decomposition of $I_{1 q}$ in contrast to the three-term breakdown of $A_{q}$ presented by Blackorby, Donaldson and Auersperg (1981), one could argue that $I_{1 q}$ is a superior cardinalization of these welfare based measures. See also Ebert (1997) who independently arrived at this single-parameter class of measures (and coincidentally calls them the "dual measures").

17 The general methodology of evaluating transformed income distributions is at the heart of several recent contributions. See for example Foster and Jin (1998), Foster and Shneyerov (1999), and the general discussion in Foster and Sen (1997).
} 


\section{Concluding remarks}

In this paper we have used a broadened form of additive decomposition to derive a new, two-parameter family of inequality measures. We have shown how the new measures relate to other well-known measures, and have identified the subclass that satisfies the transfer principle. In addition, we noted a curious duality between the measures $I_{c q}$ and $I_{q c}$ for $q \neq c$, which reveals that each measure has, in effect, two distinct decompositions.

Our main theorem generalizes a number of results in the literature, including Foster's (1983) characterization of the first Theil measure, and (in the presence of the two-person transfer principle) the results of Bourguignon (1979) and Shorrocks (1980), all of which are based on an explicit assumption of an additive decomposition. This additive form, as well as the generalized version used in this paper, can be quite useful for empirical analysis. However, the requirement that overall inequality be expressible as the sum of the between-group term and a weighted sum of subgroup inequality levels is undoubtedly rather strong, and certain milder forms of decomposition may be more tenable. For example, one might require overall inequality to be: (a) the sum of the between-group term and an alternative within-group term (as in the "path independent" requirement of Foster and Shneyerov, 1999); or (b) a general function of subgroup inequality levels and subgroup data (the "aggregability" axiom of Shorrocks, 1984); or (c) increasing in subgroup inequality levels, holding subgroup data fixed (the "subgroup consistency" axiom used by Shorrocks, 1988, and discussed extensively in Foster and Sen, 1997). In conjunction with a continuity assumption, each of these variations has been shown to lead to some subset of the $I_{c q}$ measures studied here. Further generalization along these lines may well be possible.

The axiom of general additive decomposability is based on the use of a generalized mean in evaluating subgroup prosperity levels, which clearly broadens the possibilities for decomposition analysis. However, as noted by Shorrocks (1988), this might at the same time diminish the essential distinction between withingroup and between-group inequality. For suppose that a "mean-preserving" transfer takes place entirely within one of the subgroups. One could argue that overall inequality should change only to the extent that the within-group term changes, and this is indeed the case when the arithmetic mean is employed. But since all other generalized means are influenced by such a transfer, it may well alter the between-group term of an inequality measure satisfying GAD. On the other hand, one might argue that given the key role that $\mu_{q}$ plays in the GAD approach to decomposition, it might be more appropriate to consider a " $\mu_{q}$-preserving" transfer in this thought experiment which, of course, would leave the between-group term unchanged. In any case, this potential difficulty merits further exploration.

Our final observation concerns another central tool of inequality analysis, the unanimity ordering, which identifies comparisons over which all measures in a given class agree. The Lorenz ranking, for example, is the unanimity ordering of the set of all relative inequality measure satisfying the transfer principle, which in turn may be regarded as the set of Lorenz consistent measures. Shorrocks and 
Slottje (1995) search for smaller classes that also yield the Lorenz ranking and note that the generalized entropy class is not a candidate. It would be interesting to see whether the class of Lorenz consistent $I_{c q}$ measures (which of course contains the general entropy measures) might be sufficiently discerning to signal agreement among all Lorenz consistent measures.

\section{References}

Aczél, J.: Lectures on functional equations and their applications. New York: Academic Press 1996

Aczél, J., Dombres, J.: Functional equations containing several variables. Cambridge: Cambridge University Press 1989

Anand, S.: Inequality and poverty in Malaysia. London: Oxford University Press 1983

Atkinson, A.B.: On the measurement of inequality. Journal of Economic Theory 2, 244-263 (1970)

Berge, C.: Topological spaces. New York: Macmillan 1963

Blackorby, C., Donaldson, D., Auersperg, M.: A new procedure for the measurement of inequality within and among population subgroups. Canadian Journal of Economics 14, 665-685 (1981)

Bourguignon, F.: Decomposable income inequality measures. Econometrica 47, 901-920 (1979)

Cowell, F.A.: On the structure of additive inequality measures. Review of Economic Studies 47(3), 521-531 (1980)

Cowell, F.A.: The structure of American income inequality. The Review of Income and Wealth 30, 351-375 (1984)

Cowell, F.A.: Measurement of inequality. In: Atkinson, A.B., Bourguignon, F. (eds.) The handbook of income distribution. Amsterdam: North-Holland 1998 (forthcoming)

Cowell, F.A., Jenkins, S.P.: How much inequality can we explain? A methodology and an application to the United States. Economic Journal 105, 421-430 (1995)

Cowell, F.A., Kuga, K.: Inequality measurement: an axiomatic approach. European Economic Review 15, 287-305 (1981)

Ebert, U.: Dual decomposable inequality measures. Discussion Paper V-174-97, Institut fur Volkswirtschaftslehre $I$, Universität Oldenburg (1997)

Eichhorn, W.: Measurement in economics. Heidelberg: Physica-Verlag 1988

Foster, J.E.: An axiomatic characterization of the Theil measure of income inequality. Journal of Economic Theory 65, 105-121 (1983)

Foster, J.E.: Inequality Measurement. In: Young, H.P. (ed.) Proceedings of Symposia in Applied Mathematics. Providence, R.I.: American Mathematical Society 1985

Foster, J.E., Sen, A.: On economic inequality after a quarter century. In: Sen, A. (1997)

Foster, J.E, Shneyerov, A.A.: Path independent inequality measures. Journal of Economic Theory (forthcoming) (1999)

Foster, J.E., Jin, Y.: Poverty orderings for the Dalton utility-gap measures. In: Jenkins, S.P., Kapteyn, A., Van Praag, B.M.S. (eds.) The distribution of welfare and household production. Cambridge: Cambridge University Press 1998

Foster, J.E., Ok, E.: Lorenz dominance and the variance of logarithms. Econometrica (forthcoming) (1999)

Gehrig, W.: On a characterization of the Shannon concentration measure. Utilitas Mathematica 24, 67-85 (1983)

Gehrig, W.: On the Shannon-Theil concentration measure and its characterizations. Heidelberg: Eichhorn 1988

Hardy, G.H., Littlewood, J.E., Polya, G.: Inequalities. Cambridge: Cambridge University Press 1952

Jenkins, S.P.: Accounting for inequality trends: decomposition analyses for the UK, 1971-86. Economica 65, 29-63 (1995)

Love, R., Wolfson, M.C.: Income inequality: statistical methodology and Canadian illustration. Ottawa: Statistics Canada 1976

Promislow, D.S.: An axiomatic characterization of some measures of unfairness. Journal of Economic Theory 53, 345-368 (1991)

Sen, A.: On economic inequality, enlarged edn. Oxford: Oxford University Press 1997 
Shorrocks, A.F.: The class of additively decomposable inequality measures. Econometrica 48, 613625 (1980)

Shorrocks, A.F.: Inequality decomposition by population subgroups. Econometrica 52, 1369-1385 (1984)

Shorrocks, A.F.: Aggregation issues in inequality measurement. Heidelberg: Eichhorn 1988

Shorrocks, A.F., Mookherjee, D.: A decomposition analysis of the trend in UK income inequality. Economic Journal 92, 886-902 (1982)

Shorrocks, A.F., Slottje, D.J.: Approximating unanimity orderings: an application to Lorenz dominance. Mimeo, University of Essex (1995)

Tsakloglou, P.: Aspects of inequality in Greece: measurement, decomposition and intertemporal change: 1974, 1982. Journal of Development Economics 40, 53-74 (1993) 